\title{
Effects of secondary loudspeaker properties on broadband feedforward active duct noise control
}

\author{
Yum-Ji Chan, ${ }^{a}$ Lixi Huang, and James Lam \\ Laboratory of Aerodynamics and Acoustics, HKU Zhejiang Institute of Research and Innovation, \\ Department of Mechanical Engineering, The University of Hong Kong, Pokfulam Road, Hong Kong, China
}

(Received 15 September 2012; revised 11 May 2013; accepted 16 May 2013)

\begin{abstract}
Dependence of the performance of feedforward active duct noise control on secondary loudspeaker parameters is investigated. Noise reduction performance can be improved if the force factor of the secondary loudspeaker is higher. For example, broadband noise reduction improvement up to $1.6 \mathrm{~dB}$ is predicted by increasing the force factor by $50 \%$. In addition, a secondary loudspeaker with a larger force factor was found to have quicker convergence in the adaptive algorithm in experiment. In simulations, noise reduction is improved in using an adaptive algorithm by using a secondary loudspeaker with a heavier moving mass. It is predicted that an extra broadband noise reduction of more than $7 \mathrm{~dB}$ can be gained using an adaptive filter if the force factor, moving mass and coil inductance of a commercially available loudspeaker are doubled. Methods to increase the force factor beyond those of commercially available loudspeakers are proposed. (C) 2013 Acoustical Society of America. [http://dx.doi.org/10.1121/1.4808079]
\end{abstract}

PACS number(s): 43.50.Ki, 43.38.Ja [MRB]

Pages: 257-263

\section{INTRODUCTION}

Active noise control (ANC) attempts to reduce the noise level at particular locations by generating additional, controlled sound through one or multiple secondary loudspeakers. It has been shown that ANC can attain noise reduction of over $40 \mathrm{~dB}$ if the noise is multi-harmonic, ${ }^{1}$ and broadband noise reduction of about $25 \mathrm{~dB}$ has been reported. ${ }^{2}$ Although the concept of ANC was proposed as early as in $1936,{ }^{3}$ many practical issues remain unresolved. The majority of recent ANC-related research is focused on filtering with more than a hundred papers published since 1981 (Ref. 4) to improve the efficiency $y^{5,6}$ or robustness ${ }^{7}$ adaptive filtering algorithm or its efficiency under particular situations, such as impulsive noise. ${ }^{8,9}$ New actuators designs have been tested in active noise and vibration control ${ }^{10}$ as well.

Compared with the research efforts in filter design and finding new actuators, selection criteria or the desired parameters of secondary loudspeakers in ANC is seldom discussed. In 1986, Shepherd et al. ${ }^{11}$ analyzed the power consumption of secondary loudspeakers and found that the energy efficiency of such a secondary loudspeaker is strongly frequency-dependent. Hansen ${ }^{12}$ and Lane and Clark ${ }^{13}$ proposed, respectively, novel enclosure designs and a motional feedback system to increase the low frequency output of secondary loudspeakers. To achieve an objective similar to that in ANC, Huang ${ }^{14}$ investigated the desirable mechanical impedance of a group of passive sidewall-mounted pistons leading to a high transmission loss. It was found that an ideal single sound-reflecting piston has a negative virtual mass and minimal stiffness at its support. It was proposed that such properties can be created with the aid of electromagnetic effects in addition to mechanical means but the idea

\footnotetext{
a) Author to whom correspondence should be addressed. Electronic mail: yj.chan@gmail.com
}

was not tested in that study. Later experiments ${ }^{15}$ did demonstrate the right trend but a host of practical difficulties were also identified.

Obviously the limit of noise reduction depends on the physical properties of the secondary source. By modeling a feedforward ANC system as a filtering problem in control theory, ${ }^{16}$ the broadband noise reduction is constrained mainly by the properties of the loudspeaker transfer function, which forms a part of the secondary path. Selection criteria of electrodynamic loudspeakers, the most common type of actuator in ANC, for the purpose of ANC are discussed in the current study. Based on a simple loudspeaker model, ${ }^{11}$ the dependence of three loudspeaker parameters, namely moving mass of the loudspeaker $\mathrm{M}$, suspension stiffness $\mathrm{K}$, and the force factor, $B l$, on the maximum reduction of band-pass random duct noise is investigated in the present study. Afterward, the results are interpreted in terms of characteristic frequencies of the secondary loudspeaker. Band-pass random noise is chosen because loudspeakers are ineffective actuators at extremely low frequencies, and sound waves propagated in the duct above the cut-on frequency are not planar.

Although the study is carried out in a simple system, it can illustrate principles that may be useful in other applications. We believe that these selection criteria, combined with improved loudspeaker magnet design to reduce the nonlinear behavior, ${ }^{17}$ can improve the noise reduction achieved in practice. It is envisaged that extra noise reduction can be sought by optimizing the secondary loudspeaker parameters after the current obstacles are overcome.

The main contents of this paper are organized into three sections. In Sec. II, the transfer function of a loudspeaker under various values of $B l$ are analyzed. In Sec. III, simulation in discrete time is carried out under several combinations of parameters on the secondary loudspeaker, and a similar setup is tested in experiments in Sec. IV to validate the findings. The significance of the results and possible 
ways to develop better secondary loudspeakers are discussed in Sec. IV.

The length of the finite impulse response (FIR) filter was chosen such that the magnitudes of the last steps of the FIR filter are, on average, two orders of magnitude smaller than the maximum in the filter. The Wiener filter and the filtered-x affine projection algorithm (FXAPA) are used to find the maximum noise reduction in simulations, and the latter method was adopted to find the highest noise reduction in experiments. Although the Wiener filter gives a theoretical maximum noise reduction given the transfer functions, the noise reduction achievable using an adaptive filter is relevant to the performance obtained in the experiment. FxAPA, instead of the more-popular filtered-x least mean squares (FXLMA) algorithm, is used in the experiments to avoid issues in convergence speed variation in the vicinity of the cut-off frequencies. ${ }^{18}$

\section{THEORY}

\section{A. Simple loudspeaker model}

The loudspeaker transfer function, which transforms the voltage across the loudspeaker terminals to the velocity of the loudspeaker cone, denoted $V$ and $u$, respectively, is expressed in Laplace domain in Eq. (1), ${ }^{11}$ where $B l, Z_{e}, Z_{r}$, and $Z_{m}$ denote the force factor, electrical impedance, acoustic radiation impedance, and mechanical impedance, respectively

$$
\frac{u(s)}{V(s)}=\frac{B l}{(B l)^{2}+Z_{e}(s)\left(Z_{r}(s)+Z_{m}(s)\right)} .
$$

To illustrate the effects of loudspeaker parameters on the achievable noise reduction, a simple loudspeaker model is adopted as shown in Fig. 1. A simple loudspeaker mounted on a duct wall assumes a lossless voice coil, singledegree-of-freedom mechanical vibration system (that is, the loudspeaker cone acts as a piston) and a planar sound wave traveling away from the loudspeaker. Moreover, the cavity is
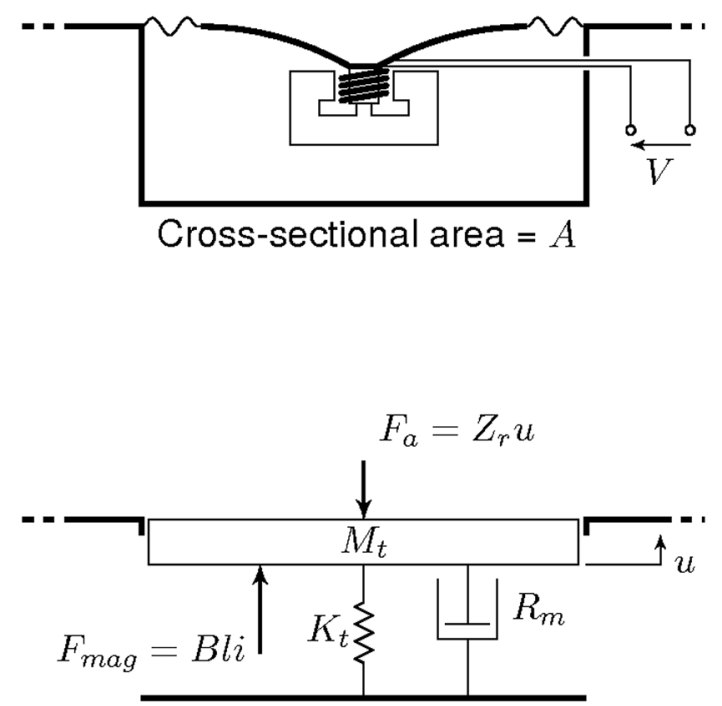

FIG. 1. Loudspeaker cavity under the simple loudspeaker model. represented by frequency-independent mass and stiffness elements. ${ }^{19}$ The simple loudspeaker model, which does not take into account of magnetic losses at high frequencies ${ }^{20}$ and non-linear behavior, ${ }^{21}$ allows the loudspeaker transfer function to be written as a rational function.

The electrical, mechanical, and radiation impedances can be expressed in terms of physical parameters explained in Table I:

$$
\begin{aligned}
& Z_{e}(s)=R_{e}+s L_{e}, \\
& \begin{aligned}
Z_{m}(s) & =\left(M+\frac{\rho d A}{3}\right) s+R_{m}+\frac{1}{s}\left(K+\frac{\rho c^{2} A}{d}\right) \\
& =M_{t} s+R_{m}+\frac{1}{s} K_{t},
\end{aligned} \\
& Z_{r}=\frac{1}{2} \rho c A .
\end{aligned}
$$

The imaginary part (near field) of the radiation impedance $^{14}$ may be lumped with the mechanical mass of the loudspeaker, and this will not alter the conclusions reached in this study. In addition to the parameters listed in Table I, Substituting Eq. (2) into Eq. (1) gives

$$
\frac{u(s)}{V(s)}=\frac{B l s}{s(B l)^{2}+\left(s L_{e}+R_{e}\right)\left(M_{t} s^{2}+R_{t} s+K_{t}\right)},
$$

where $R_{t}=R_{m}+Z_{r}$. Equation (3) is analyzed in the next section by looking at its poles.

\section{B. Selected loudspeaker transfer functions and their corresponding ideal filters in ANC}

Throughout the discussion, the real pole of the transfer function in Eq. (3) is denoted as $-p$ and, for the conjugate pair of poles, $\left(\sigma+j \omega_{t}\right)$ and $\left(\sigma-j \omega_{t}\right)$ where $\omega_{t}>0$. To maintain stability, $p>0$ and $\sigma<0$. The values of $p$ and $\omega_{t}$ are called characteristic frequencies in the following text. The loudspeaker transfer function can be written in terms of $\mathrm{p}$, $\omega_{t}$, and $\sigma$ as shown in Eq. (4)

TABLE I. Nomenclature of physical parameters and values chosen in the base system.

\begin{tabular}{llcc}
\hline \hline Parameter & \multicolumn{1}{c}{ Description } & Value & Unit \\
\hline$K$ & Suspension stiffness of cone & 3695 & $\mathrm{Nm}^{-1}$ \\
$M$ & Moving mass & 6.5 & $\mathrm{~g}$ \\
$R_{m}$ & Mechanical damping coefficient & 1.67 & $\mathrm{~kg} \cdot \mathrm{s}^{-1}$ \\
$A$ & Cross section area of the duct & 0.0143 & $\mathrm{~m}^{2}$ \\
$\rho$ & Density of air & 1.20 & $\mathrm{~kg} \cdot \mathrm{m}^{-3}$ \\
$c$ & Speed of sound in air & 340 & $\mathrm{~ms}$ \\
$B l$ & Force factor & 5.7 & $\mathrm{~T} \cdot \mathrm{m}$ \\
$R_{e}$ & Resistance of voice coil & 6.3 & $\Omega$ \\
$L_{e}$ & Inductance of voice coil & 0.7 & $\mathrm{mH}$ \\
$d$ & Depth of the back cavity & 0.1 & $\mathrm{~m}$ \\
$K_{t}$ & Effective stiffness & 23484 & $\mathrm{Nm}$ \\
$R_{t}$ & Effective damping coefficient & 4.58 & $\mathrm{~kg} \cdot \mathrm{s}^{-1}$ \\
$M_{t}$ & Effective mass & 7.07 & $\mathrm{~g}$ \\
\hline \hline
\end{tabular}




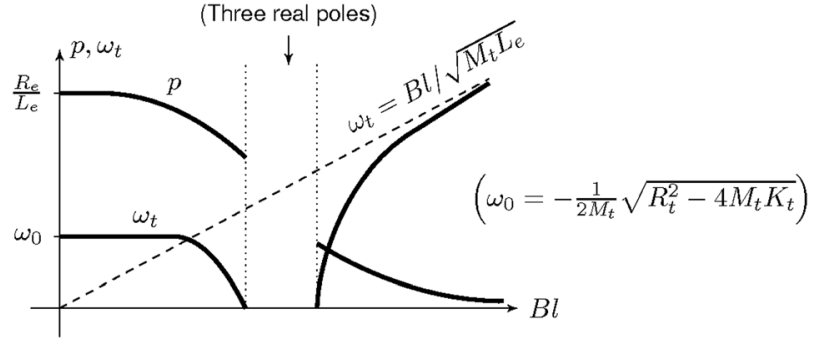

FIG. 2. Sketch of characteristic frequencies of loudspeaker with varying $B l$.

$$
\frac{u(s)}{V(s)}=\frac{B l}{p L_{e} M_{t}} \frac{1}{(1+s / p)} \frac{s}{\left(s-\sigma-j w_{t}\right)\left(s-\sigma+w_{t}\right)} .
$$

If a loudspeaker system has a small force factor (that is, $B l \rightarrow 0)$, the value of $(B l)^{2}$ in the denominator of Eq. (3) is negligible, and the effects of electrical and mechanical parameters in a loudspeaker system can be separated: The real pole $p$ in such a system is determined solely by $Z_{e}\left(p=R_{e} /\right.$ $L_{e}$ ) and the values of $\sigma$ and $\omega_{t}$ are determined by mechanical parameters and radiation impedance. The parameters $p, \omega_{t}$, and $B l$ given a set of mechanical and electrical parameters is related as shown in the sketch in Fig. 2.

The Bode magnitude plot of the loudspeaker transfer function can be sketched based on the characteristic frequencies if $p$ and $\omega_{t}$ are far apart with $s=j \Omega$. On one hand, Fig. 2 shows that $p \gg \omega_{t}$ with typical loudspeaker parameters and a small value of $B l$, and the corresponding Bode magnitude plot is sketched in Fig. 3. In addition, if any frequency in the passband, denoted $\Omega$, satisfy $\omega_{t} \ll \Omega \ll$ p, Eq. (4) can be approximated as

$$
\frac{u(j \Omega)}{V(j \Omega)}=\left(\frac{B l}{p L_{e} M_{t}}\right) \cdot 1 \cdot \frac{1}{j \Omega},
$$

which is an integrator.

On the other hand, it is shown in Fig. 2 that a high value of $B l$ leads to $p \ll \omega_{t}$, and the corresponding Bode magnitude plot is sketched in Fig. 4. In the extreme case with $B l$ $\rightarrow \infty, u / \mathrm{V} \approx 1 /(B l)$.

It is noted that the magnitude of $B l$ in the discussion in the preceding text is relative. The noise reduction improvements by increasing $B l$ can be achieved by reducing $K_{t}, R_{t}$, and $M_{t}$, which involve the loudspeaker parameters as well as the size of the cavity. According to Eq. (3), the noise reduction of a system with $K_{t}, R_{t}$, and $M_{t}$ scaled by a factor of $\alpha$

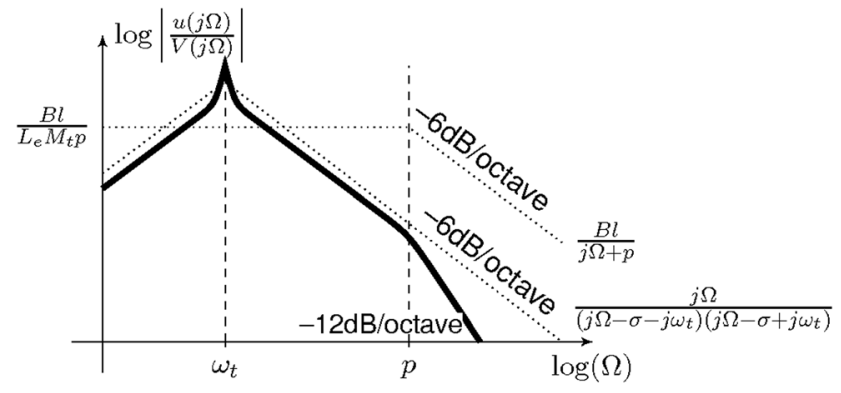

FIG. 3. Bode magnitude sketch of loudspeaker transfer function with $p \gg \omega_{t}$.

would be equivalent to a counterpart with force factor $B l$ scaled by $\sqrt{1 / \alpha}$.

The ideal filter in feedforward active noise control is

$$
W_{i}(j \Omega)=\frac{P(j \Omega)}{S(j \Omega)},
$$

where $P(j \Omega)$ is the transfer function from the reference signal to the signal captured at the error microphone (also known as the primary path) and $S(j \Omega)$ is the secondary path, which is the transfer function from the signal fed into the secondary loudspeaker to the signal captured at the error microphone. The secondary path can be simplified as the cascade of the loudspeaker transfer function and a time delay of $\tau$, which represents the time for the sound wave traveling from the secondary loudspeaker to the error microphone

$$
S(j \Omega)=\frac{u(j \Omega)}{V(j \Omega)} \underbrace{\frac{p(j \Omega)}{u(j \Omega)}}_{Z_{r}} e^{-j \tau \Omega} .
$$

Given a simple loudspeaker model, the ideal filter for active noise control under very small and very large force factors can be approximated, if the passband of the noise is located entirely between $p$ and $\omega_{t}$. The ideal filter under $p \gg \omega_{t}$ can be approximated by substituting Eq. (5) into Eq. (7)

$$
W_{i}(j \Omega) \approx-j \Omega \frac{2 p L_{e} M_{t}}{B l \rho c A} e^{j \tau \Omega} P(j \Omega),
$$

which is the product of $P(s)$, a differentiator and a time advance. The time advance denoted $e^{j \tau \Omega}$ is usually compensated by a longer time delay in $P(j \Omega)$. Alternatively, if $p \ll \omega_{t}$ (for example, if $B l$ is large),

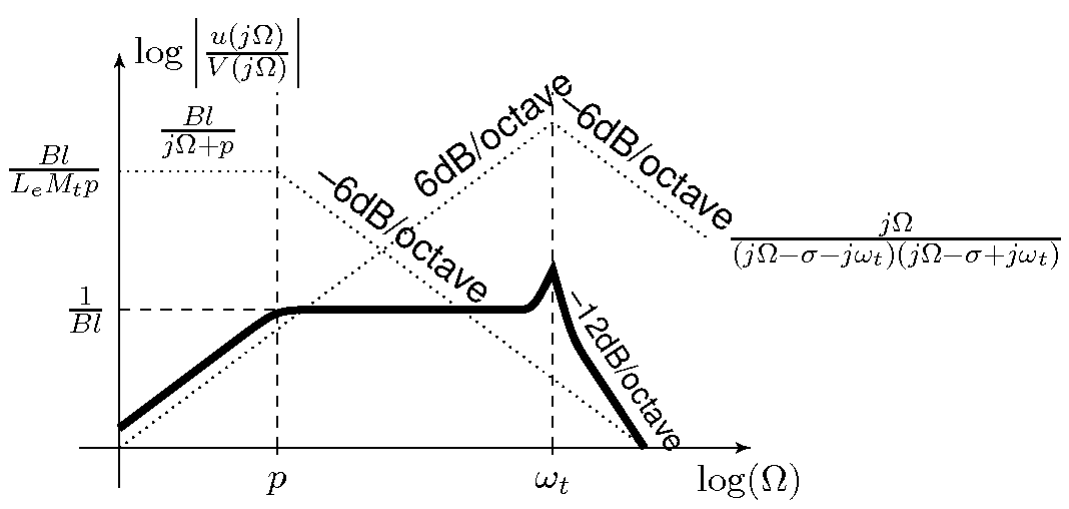

FIG. 4. Bode magnitude sketch of loudspeaker transfer function with $p \ll \omega_{t}$. 


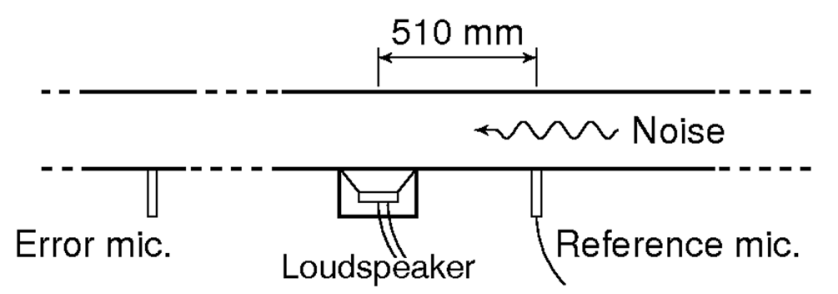

FIG. 5. System modeled in the simulation.

$$
W_{i}(j \Omega) \approx-\frac{2 B l}{\rho c A} e^{j \tau \Omega} P(j \Omega) .
$$

Besides the two specific cases shown in Eq. (8) and (9), $W_{i}(j \Omega)$ is impossible to be implemented because the loudspeaker transfer function [Eq. (3)] is strictly proper.

\section{SIMULATION}

The maximum feedforward active noise reduction under various combinations of secondary loudspeaker parameters was sought in discrete-time numerical model. The schematic of the modeled system is shown in Fig. 5. In the model, the secondary loudspeaker with a back cavity was installed on the duct wall. The effective cone area of the loudspeaker is assumed to be equal to the cross-sectional area of the duct. The loudspeaker and cavity, forming $u(j \Omega) / V(j \Omega)$ in Eq. (7), is modeled as an infinite impulse response (IIR) filter of order 3 . The time delay in the secondary path between the loudspeaker and the error microphone is compensated by the identical delay in the primary path.

The reference microphone was located $510 \mathrm{~mm}$ upstream of the secondary loudspeaker center, and the contribution from the secondary loudspeaker to the signal captured at the reference microphone was removed. The band-pass random noise fed into the system from the far end has a flat passband with cutoff frequencies of 150 and $1050 \mathrm{~Hz}$. A high sampling frequency of $20 \mathrm{kHz}$ was adopted to avoid phase distortion near to the Nyquist frequency. The parameters of the base system, which are drawn from one of the loudspeakers used in experiments, are listed in Table I. By substituting the values of the parameters into Eq. (3), $p=8172, \omega_{t}=1765$, and $\sigma=737.5$. As the values of $p$ and $\omega_{t}$ of the base loudspeaker system are similar to those in $B l \rightarrow 0$ (where $p_{0}=9000$ and

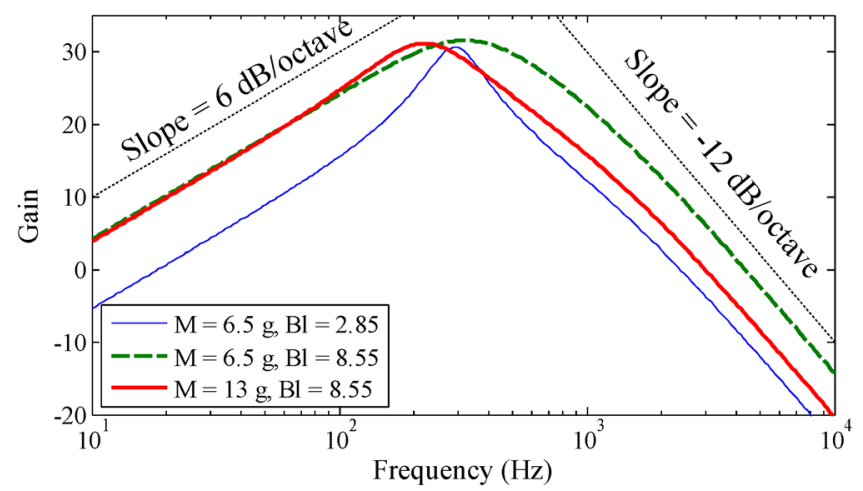

FIG. 6. (Color online) Bode magnitude plots of selected loudspeaker systems.
TABLE II. Characteristic frequencies of selected loudspeaker systems.

\begin{tabular}{lccc}
\hline \hline$B l$ & 2.85 & 8.55 & 8.55 \\
$K\left(\mathrm{Nm}^{-1}\right)$ & 3695 & 3695 & 3695 \\
$M(\mathrm{~g})$ & 6.5 & 6.5 & 13 \\
$p / 2 \pi(\mathrm{Hz})$ & 1402 & 1077 & 1277 \\
$\omega_{t} / 2 \pi(\mathrm{Hz})$ & 286 & 244 & 196 \\
Noise reduction $(\mathrm{dB})$ & 29.5 & 33.8 & 36.2 \\
\hline \hline
\end{tabular}

$\left.\omega_{t 0}=1794\right)$, the force factor of the base loudspeaker system can be considered small, and the characteristic frequencies are mainly determined by the mechanical parameters of the loudspeaker and the radiation impedance on the loudspeaker cone.

One or two of the parameters $B l, K$, and $M$ in the secondary loudspeaker system were perturbed at the same time and the broadband noise reduction achievable was observed. The loudspeaker moving mass $(M)$ and the suspension stiffness $(K)$ varied by up to factors of 2 and 4 , respectively, and $B l=2.85,5.7$, or $8.55 \mathrm{~T} \cdot \mathrm{m}$. Bode magnitude plots of three selected loudspeaker systems, the characteristic frequencies of which are listed in Table II, are shown in Fig. 6. It is noted that the force factors of 2.85 or $5.7 \mathrm{~T} \cdot \mathrm{m}$ can be considered small in perturbed systems because (1) $\omega_{t}$ increases with rising $\sqrt{K_{t} / M_{t}}$ and (2) $p$ is close to (or much higher) than the upper frequency limit of the passband. This assumption does not hold if $B l=8.55 \mathrm{~T} \cdot \mathrm{m}$ : In fact, $p<\omega_{t}$ with $B l=8.55 \mathrm{~T} \cdot \mathrm{m}$, $K=3695 \mathrm{Nm}^{-1}$, and $M=3.25 \mathrm{~g}$.

In the first step, 800-tap optimal FIR filter was sought for each configuration using Wiener filter. The autocorrelation matrix and the cross-correlation vector were obtained using the unit pulse responses of the bandpass filter and the loudspeaker transfer function where appropriate. The conjugate gradient method was used to solve the Toeplitz system ${ }^{22}$ because the autocorrelation matrices of band-pass random noise have poor condition numbers. It can be seen that broadband feedforward active noise control noise reduction of up to $53 \mathrm{~dB}$ can be achieved using Wiener filters. Although the variation among the data in Table III is small, the results show a clear trend that a higher value of $B l$ leads to improved noise reduction. By increasing $\mathrm{Bl}$ by $50 \%$, the noise reduction can improve by up to $1.6 \mathrm{~dB}$. The noise reduction improvement can be explained by comparing the acoustic, mechani$\mathrm{cal}$, and electromagnetic forces acting on the loudspeaker cone. Acoustic force is determined by radiation impedance $Z_{r}$ with $F_{a}=Z_{r} u$, and the acoustic impedance is independent of the loudspeaker parameters except the cross section area of the cone. Mechanical forces are related to the mechanical parameters of the loudspeaker moving parts, and the

TABLE III. Noise reduction achieved (in $\mathrm{dB}$ ) using the Wiener filter.

\begin{tabular}{lccccccc}
\hline \hline$K\left(\mathrm{Nm}^{-1}\right)$ & 3695 & $\mathbf{9 2 3 . 8}$ & $\mathbf{1 8 4 8}$ & $\mathbf{7 3 9 0}$ & $\mathbf{1 4 7 8 0}$ & 3695 & 3695 \\
$M(\mathrm{~g})$ & 6.5 & 6.5 & 6.5 & 6.5 & 6.5 & $\mathbf{3 . 2 5}$ & $\mathbf{1 3}$ \\
$B l=2.85$ & 56.2 & 58.2 & 56.4 & 56.4 & 56.3 & 56.2 & 56.4 \\
$B l=5.7$ & 58.2 & 58.9 & 57.5 & 58.4 & 57.6 & 58.4 & 58.4 \\
$B l=8.55$ & 58.8 & 60.3 & 58.2 & 59.0 & 59.2 & 58.9 & 58.5 \\
\hline \hline
\end{tabular}


TABLE IV. Noise reduction achieved (in dB) using FXAPA.

\begin{tabular}{lccccccccc}
\hline \hline$K\left(\mathrm{Nm}^{-1}\right)$ & 3695 & $\mathbf{9 2 3 . 8}$ & $\mathbf{1 8 4 8}$ & $\mathbf{7 3 9 0}$ & $\mathbf{1 4 7 8 0}$ & 3695 & 3695 & 3695 & 3695 \\
$M(\mathrm{~g})$ & 6.5 & 6.5 & 6.5 & 6.5 & 6.5 & $\mathbf{3 . 2 5}$ & $\mathbf{4 . 8 8}$ & $\mathbf{9 . 7 5}$ & $\mathbf{1 3}$ \\
$B l=2.85$ & 29.5 & 30.4 & 30.1 & 28.4 & 26.8 & 28.2 & 28.9 & 30.3 & 31.2 \\
$B l=5.7$ & 31.7 & 32.6 & 32.3 & 30.7 & 29.1 & 30.3 & 31.0 & 32.8 & 34.0 \\
$B l=8.55$ & 33.8 & 34.6 & 34.3 & 32.9 & 31.5 & 32.7 & 33.3 & 34.9 & 36.2 \\
\hline
\end{tabular}

electromagnetic force acting on the loudspeaker cone is equal to $F_{e m}=B l i$, with $i$ being the current passing through the voice coil. If the force factor is high, the total force acting on the loud-speaker cone is dominated by the magnetic force, thus the velocity is roughly proportional to the voltage applied across the terminals and the transfer function is flat within a wide frequency band (Fig. 4).

Afterward, each configuration is re-run using a fifth-order FXAPA (Ref. 23) to investigate the noise reduction performance using adaptive filtering and to predict the noise reduction obtainable in experiments. The length of FIR filters is maintained at 800 , and the noise reduction was measured by the residual generated by the resultant FIR filter as if the filter was fixed. The improvement of noise reduction with increased $B l$ (2.1 dB increment with $50 \%$ increase in $B l$ ) is more evident as shown in Table IV. In addition, the broadband noise reduction is improved by $1.1 \mathrm{~dB}$ if the moving mass is $50 \%$ higher than the base system. The Wiener and FFXAPA results are different because a random noise signal is fed to the FXAPA while the random input is simulated using the autocorrelation matrix of the bandpass filter unit pulse response in the Wiener counterpart. In addition, while the convergence of FXAPA is generally believed to be superior to filtered-X-normalized-least mean squares (FXNLMS) algorithm, the exact behavior is still under research. ${ }^{24}$

The broadband noise reduction obtained using FXAPA is apparently insensitive to changes in the suspension stiffness of the secondary loudspeaker because the effective stiffness of a 100-mm-deep cavity is $19790 \mathrm{Nm}^{-1}$, which is more than 5 times of the suspension stiffness $K$. As a result, the total effective stiffness $\left(K_{t}\right)$ is insensitive to $K$. If the value of $K_{t}$ is halved compared with the base system, the

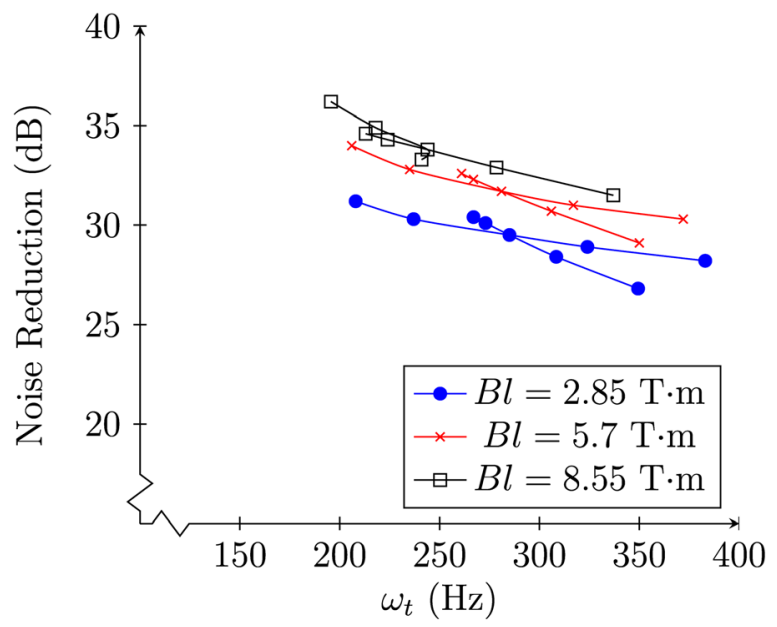

FIG. 7. (Color online) Relationship between $\omega_{t}$ and maximum noise reduction obtained using FXAPA in systems where $p>\omega_{t}$.
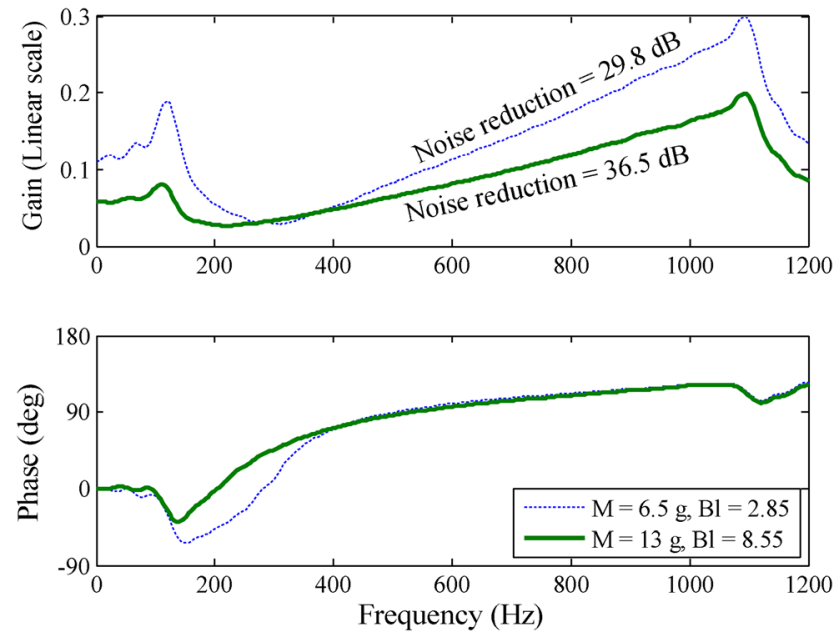

FIG. 8. (Color online) Bode plot of $W(s)$ obtained using FXAPA without time delay component.

noise reduction achievable under FXAPA is improved by $5.4 \mathrm{~dB}$. Although a reduced $K_{t}$ can lead to extra noise reduction, practical concerns have to be considered. As $K_{t}$ is dominated by the stiffness of air in the cavity, reduced $K_{t}$ refers to a deeper cavity, but approximations as simple stiffness and mass elements become invalid.

The improvement in noise reduction obtained using FXAPA can be predicted by observing the reduction of $\omega_{t}$. In systems with a small $B l$, this occurs at an increased moving mass or a reduced stiffness. By doubling the moving mass $(M)$ and increasing the force factor $(B l)$ by $50 \%$ at the same time, the noise reduction achievable is increased by $4.5 \mathrm{~dB}$. With the value of $p$ insensitive to the mechanical parameters, a larger portion of the passband sits between the two characteristic frequencies if $\omega_{t}$ is reduced. Noise reduction achieved in the perturbed systems is plotted against $\omega_{t}$ in Fig. 7. If $\omega_{t}$ and $p$ are far apart, the transfer function of the system in the passband fits better to the approximation shown in Eq. (5), where an ideal filter [Eq. (8)] exists to lead to perfect noise cancellation. The transfer function of selected systems and of the filters given by FXAPA for these systems (without the time-delay component) are plotted in Figs. 6 and 8, respectively.

\section{EXPERIMENTAL STUDIES}

Experiments were carried out to validate two findings in Sec. III that the noise reduction performance can be improved (1) by increasing the force factor $(B l)$ or (2) by increasing the moving mass $(M)$. The experiments were carried out in a square duct with 140 -mm cross section illustrated in Fig. 9, where the key longitudinal dimensions are annotated. The average depth of the loudspeaker cavity is $90 \mathrm{~mm}$. Active noise control was performed with the aid of a dSPACE ds 1006 controller with a sampling rate of $12.5 \mathrm{kHz}$.

The block diagram of the experiment is shown in Fig. 10. Weighted band-pass random signal with cut-off frequencies 120 and $1050 \mathrm{~Hz}$ was fed across the terminals of the primary loudspeaker, such that the power spectral density of noise at the error microphone has a flat passband. The primary path $P(z)$ can be treated as a 150 step-delay (or $12 \mathrm{~ms}$ 


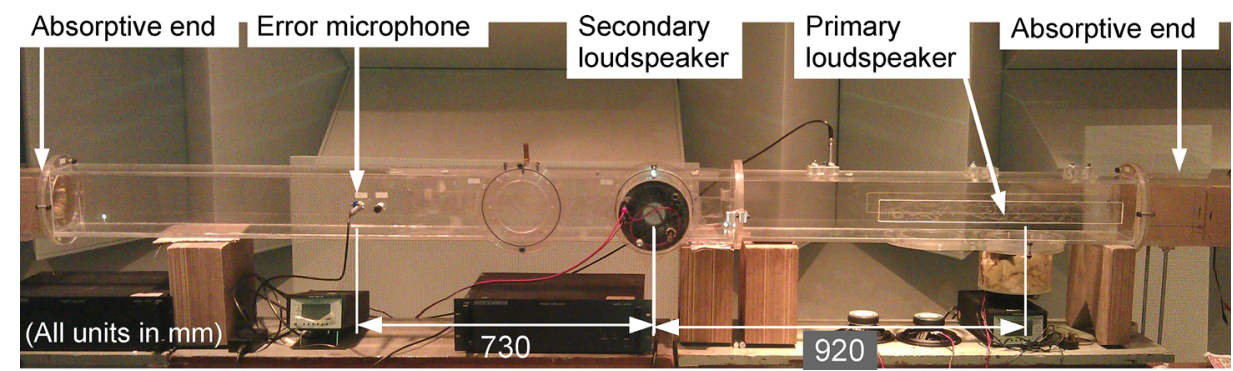

FIG. 9. (Color online) Duct used in active noise control experiment.

at a sampling rate of $12.5 \mathrm{kHz}$ ). The secondary path denoted $S(z)$ is the transfer function from the signal fed into the secondary loudspeaker to that captured at the error microphone. In the experiments, the secondary path is modeled as a 1000tap FIR filter.

Similar to the approach taken in simulation, a seventhorder FXAPA is used to find an optimal 400-tap FIR filter to minimize the sound pressure level at the error microphone. Each test lasted $25 \mathrm{~s}$, of which the adaptive filter was turned on $1.5 \mathrm{~s}$ later than the noise source in order to measure the sound pressure level without ANC. The experiment was repeated in each configuration with increasing normalized adaptation constant $(\tilde{\mu})$ until the system became unstable.

The parameters of the chosen secondary loudspeakers, as provided by the manufacturer, are compared in Table V. Loudspeakers A and B have similar mechanical and electrical parameters but the force factor $(B l)$ of Loudspeaker $A$ is less than half of that of Loudspeaker B. To increase the moving masses of Loudspeakers A and B, Blu-tack adhesive of $2.71 \mathrm{~g}$ and $2.92 \mathrm{~g}$, respectively, were attached at the back of the loudspeaker cones.

The noise reduction measured at the error microphone over time is plotted in Fig. 11. The experimental results validate the simple loudspeaker model and the findings in the simulations, as the noise reduction performance is $0.4-3.5 \mathrm{~dB}$ higher by using a loudspeaker with a larger force factor, $\mathrm{Bl}$. In addition, it was found that a higher value of the adaptation constant $\left(\tilde{\mu}=(12-15) \times 10^{-4}\right.$ is allowed with Loudspeaker B than if Loudspeaker $\mathrm{A}$ is used [where $\tilde{\mu}=(6-8) \times 10^{-4}$ ], thus convergence of the adaptive algorithm is quicker. The noise reduction with using Loudspeaker B as the secondary loudspeaker is improved from 31.3 to $32.5 \mathrm{~dB}$ by installing the additional mass. However, the noise reduction performance deteriorates with added mass to Loudspeaker A $(30.9 \mathrm{~dB}$ to $29.1 \mathrm{~dB})$. The discrepancy between the experimental results and the theoretical prediction may come from the imbalance of mass in the loudspeaker cone, which is not considered in the simple loudspeaker model. It is found in the spectral densities of the error signal (Fig. 12) that the improvement in noise reduction using a secondary loudspeaker with a larger value of $B l$ is more evident above $600 \mathrm{~Hz}$.

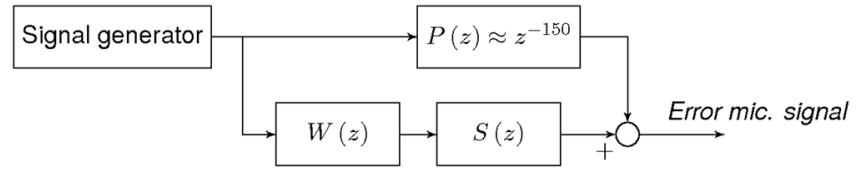

FIG. 10. Block diagram of the experiment.
TABLE V. Parameters of secondary loudspeakers used in experiments.

\begin{tabular}{lcccc}
\hline \hline Parameter & Description & Speaker A & Speaker B & Unit \\
\hline$K$ & Suspension stiffness of cone & 1310 & 3695 & $\mathrm{Nm}^{-1}$ \\
$M$ & Moving mass & 5.9 & 6.5 & $\mathrm{~g}$ \\
$R_{m}$ & Mechanical viscous damping & 0.563 & 1.667 & $\mathrm{~kg} \cdot \mathrm{s}^{-1}$ \\
$R_{e}$ & Resistance of voice coil & 6.8 & 6.3 & $\Omega$ \\
$L_{e}$ & Inductance of voice coil & 0.8 & 0.7 & $\mathrm{mH}$ \\
$B l$ & $\quad$ Force factor & 2.5 & 5.7 & $\mathrm{~T} \cdot \mathrm{m}$ \\
& Lower frequency limit & 75 & 120 & $\mathrm{~Hz}$ \\
$A$ & Effective piston area & & 0.0143 & $\mathrm{~m}{ }^{2}$ \\
$\rho c$ & Specific acoustic impedance & 407 & $\mathrm{Nm}{ }^{-3} \cdot \mathrm{s}$ \\
$d$ & Nominal depth of back cavity & & 0.1 & $\mathrm{~m}$ \\
$r_{c}$ & Diameter of back cavity & & 0.1 & $\mathrm{~m}$ \\
\hline \hline
\end{tabular}

(Speaker A: VISATON FR 6.5-8 $\Omega$, Speaker B: VISATON BG17-8 $\Omega$ ).

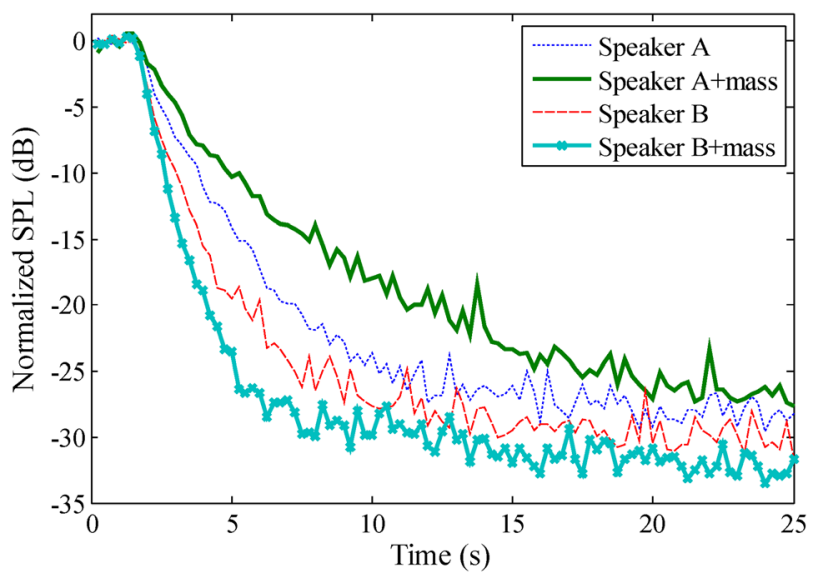

FIG. 11. (Color online) Sound pressure level at error microphone.

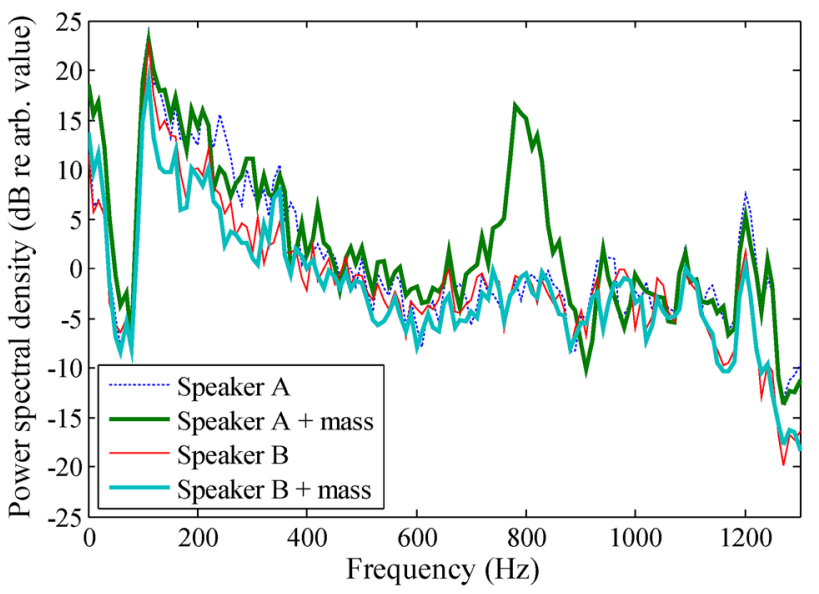

FIG. 12. (Color online) Frequency spectra of error microphone signal. 


\section{A. Prospect of further improvements}

Simulation results show that noise reduction can be gained by increasing the force factor by $50 \%$ above that provided by speaker B (i.e., $B l=5.7 \mathrm{~T} \cdot \mathrm{m}$ ). However, it is challenging to increase the force factor $B l$ in practice. In theory, the force factor $B l$ is the product of flux density, $B$, and the length of the coil wire, $l$. On one hand, it was proposed to increase $B$ by filling the air gap between the magnet and voice coil with ferrofluid, ${ }^{25}$ but the flux density in a magnet cannot be increased indefinitely because many common magnetic materials saturate between $1.0 \mathrm{~T}$ and $1.4 \mathrm{~T}^{26} \mathrm{On}$ the other hand, if the coil diameter is kept constant, the total moving mass $M$, electrical resistance $R_{e}$, and inductance $L_{e}$ are proportional to the length of the coil wire, $l$. Suppose $B l$, $M$, and $L_{e}$ of the base loudspeaker system are doubled simultaneously and the increased electrical resistance across the coil terminals are compensated by employing negative impedance converters (NICs), ${ }^{27}$ the broadband noise reduction in simulation is improved from 31.7 to $39.0 \mathrm{~dB}$ with FXAPA, which is an improvement of over $7 \mathrm{~dB}$.

\section{CONCLUSIONS}

Dependence of feedforward active duct noise control performance on selected properties of the secondary loudspeaker were investigated by using a simple loudspeaker model and experiment. In a typical case with commercially available loudspeakers installed with back cavities, simulation results show that the most desirable design change is by increasing the force factor $B l$ because it will lead to better noise reduction and quicker convergence speed. This is because the movement of the loudspeaker cone in these loudspeakers are dominated by the electromagnetic forces. Methods to increase force factor $B l$ further, which is related to the flux density and the wire length of the voice coil, have been proposed. The noise reduction is also improved by having a heavier moving mass or a lower suspension stiffness.

\section{ACKNOWLEDGMENTS}

The postdoctoral research fellowship of the first author is supported by the University of Hong Kong and Research Grants Council (HKRGC) Project No. GRF 7138/10E. Support from a China National Key Basic Research Scheme (2012CB7202) is also acknowledged.

${ }^{1} \mathrm{X}$. Guo and M. Bodson, "Analysis and implementation of an adaptive algorithm for the rejection of multiple sinusoidal disturbances," IEEE Trans. Control Syst. Technol. 17, 40-50 (2009).
${ }^{2}$ K. Fujii, K. Yamaguchi, S. Hashimoto, Y. Fujita, and M. Muneyasu, "Verification of simultaneous equations method by an experimental active noise control system," Acoust. Sci. Technol. 27, 270-277 (2006).

${ }^{3}$ P. Lueg, "Process of silencing sound oscillation," U.S. patent 2,043,416 (1936).

${ }^{4} \mathrm{~J}$. C. Burgess, "Active adaptive sound control in a duct: A computer simulation,” J. Acoust. Soc. Am. 70, 715-726 (1981).

${ }^{5}$ S. C. Douglas, "An efficient implementation of the modified filtered-x LMS algorithm," IEEE Signal Process. Lett. 4, 286-288 (1997).

${ }^{6}$ D. H. Crawford and R. W. Stewart, "Adaptive IIR filtered-v algorithms for active noise control," J. Acoust. Soc. Am. 101, 2097-2103 (1997).

${ }^{7}$ M. Akhtar and W. Mitsuhashi, "Improving performance of hybrid active noise control systems for uncorrelated narrowband disturbances," IEEE Trans. Audio, Speech, Lang. Process. 19, 2058-2066 (2011).

${ }^{8}$ N. Han, S. Feng, and X. Qiu, "Active control of one-dimension impulsive reflection based on a prediction method," J. Acoust. Soc. Am. 127, 1193-1196 (2010).

${ }^{9}$ M. T. Akhtar and W. Mitsuhashi, "Improving performance of FxLMS algorithm for active noise control of impulsive noise," J. Sound Vib. 327, 647-656 (2009).

${ }^{10} \mathrm{M}$. Cuesta, P. Cobo, A. Fernábaéndez, and J. Pfretzschner, "Using a thin actuator as secondary source for hybrid passive/active absorption in an impedance tube," Appl. Acoust. 67, 15-27 (2006).

${ }^{11}$ I. Shepherd, A. Cabelli, and R. LaFontaine, "Characteristics of loudspeakers operating in an active noise attenuator," J. Sound Vib. 110, 471-481 (1986).

${ }^{12}$ C. H. Hansen, Understanding Active Noise Cancellation (Spon, London, 2001), pp. 72-73.

${ }^{13}$ R. L. Lane and S. A. Clark, "Improving loudspeaker performance for active noise control applications," J. Audio Eng. Soc. 46, 508-519 (1998).

${ }^{14}$ L. Huang, "A theory of reactive control of low-frequency duct noise," J. Sound Vib. 238, 575-594 (2000).

${ }^{15}$ Y. H. Chiu, L. Cheng, and L. Huang, "Drum-like silencers using magnetic forces in a pressurized cavity," J. Sound Vib. 297, 895-915 (2006).

${ }^{16}$ A. Saberi, A. A. Stoorvogel, and P. Sannuti, Filtering Theory: With Applications to Fault Detection, Isolation and Estimation (Birkhäuser, Boston, 2007), pp. 712.

${ }^{17}$ B. Merit, G. Lemarquand, and V. Lemarquand, "In pursuit of increasingly linear loudspeaker motors," IEEE Trans. Magnet. 45, 2867-2870 (2009).

${ }^{18} \mathrm{D}$. Zhou and V. DeBrunner, "A new active noise control algorithm that requires no secondary path identification based on the SPR property," IEEE Trans. Signal Process. 55, 1719-1729 (2007).

${ }^{19}$ L. E. Kinsler, A. R. Frey, A. B. Coppens, and J. V. Sanders, Fundamentals of Acoustics, 3rd ed. (Wiley and Sons, New York, 1982), pp. 200-202.

${ }^{20} \mathrm{~W}$. M. Leach, Jr., "Loudspeaker voice-coil inductance losses: Circuit models, parameter estimation, and effect on frequency response," J. Audio Eng. Soc 50, 442-450 (2002).

${ }^{21}$ R. Ravaud, G. Lemarquand, V. Lemarquand, and T. Roussel, "Ranking of the nonlinearities of electrodynamic loudspeakers," Arch. Acoust. 35, 49-66 (2010).

${ }^{22}$ R. H. Chan and M. K. Ng, "Conjugate gradient methods for toeplitz systems," SIAM Rev. 38, 427-482 (1996).

${ }^{23}$ Least-Mean-Square Adaptive Filters, edited by S. Haykin and B. Widrow, (Wiley, Hoboken, NJ, 2003), pp. 241-292.

${ }^{24}$ T. Paul and T. Ogunfunmi, "On the convergence behavior of the affine projection algorithm for adaptive filters," IEEE Trans. Circuits and Syst., I: Regul. Pap. 58, 1813-1826 (2011).

${ }^{25}$ S. Tsuda and R. E. Rosensweig, "Ferrofluid centered voice coil speaker," U.S. Patent 7,729,504 (2010).

${ }^{26} \mathrm{~K}$. Strnat, "Modern permanent magnets for applications in electro-technology," Proc. IEEE 78, 923-946 (1990).

${ }^{27}$ R. Schaumann, H. Xiao, and M. E. van Valkenburg, Design of Analog Filters, 2nd ed. (Oxford University Press, New York, 2010), pp. 594-600. 